\title{
Adolescents' Sexual and Reproductive Health Challenges in Northern Nigeria: Road Map to Effective Interventions
}

\author{
James Godswill \\ Department of Sociology, Kaduna State University, Tafawa Balewa Way, Kaduna, Nigeria \\ Phone: +2348037030868 \\ E-mail address: jgodswill13@gmail.com
}

\begin{abstract}
Young people have been well documented as special need group in the area of reproductive health. Adolescent sexuality and reproductive health are important contemporary concerns especially for reproductive health problems such as early marriage, unintended/unwanted pregnancy, maternal mortality and sexually transmitted diseases, including AIDS. There are yet many unmet reproductive health needs for the African adolescents despite the many reproductive health programmes which have been initiated in the last decade. Hence, this study examines the challenges of adolescents' sexual and reproductive health in northern Nigeria. Primary data were obtained using qualitative methods. Thirtysix focus group discussions (FGDs) were conducted among adolescents aged 15-19 years, stratified by sex, marital status, fertility status and residence; and 48 in-depth interviews (IDIs) with communityresident adolescents who have ever married or/and have begun childbearing. Results show that teenage marriage and childbearing is a highly cherished activity among adolescents in northwestern Nigeria, consequently their attitudes were largely pronatalist. Knowledge of contraceptive methods clearly exists but gender-biased, even though contraceptive approval and use was low. Sense of vulnerability and fear clearly exist as a number of participants expressed lack of courage to confront appropriate authorities, including their parents with reproductive health issues. Motivations for teenage marriage and childbearing reflect various levels of influence, such as parental pressure and social norms, individual needs, including socio-economic survival. There is need for intervention strategies to tackle issues relating to empowerment, and access to resources, improved information and services.
\end{abstract}

Keywords: Reproductive health; Sexuality; Northern Nigeria; Adolescent; Health Programme

\section{INTRODUCTION}

Reproductive health has been defined as a state of complete social, physical and physiological well-being in all matters of reproductive system (Shane, 1992). This will include the social and medical support that enables women go through pregnancy and childbirth. Similarly, sexual health is not restricted to the act of having sex, but refers to "a state of physical, emotional, mental and social well being in relation to sexuality and not merely the absence of disease, dysfunction or infirmity". Sexual health requires a positive and respectable approach to sexuality and sexual relationships, as well as the possibility of having pleasurable and safe sexual experiences free from coercion, discrimination and violence. For sexual health 
to be attained and maintained in any social setting, the sexual rights of all persons must be respected, protected and fulfilled. Sexual rights necessitate that all persons, irrespective of sex, are free from coercion, discrimination and violence, to the highest attainable standard of sexual health, including access to sexual and reproductive health care services; seek, receive and impart information related to sexuality; sexuality education; respect for bodily integrity; choose their partner; decide to be sexually active or not; consensual sexual relations; consensual marriage; decide whether or not, and when, to have children; and pursue a satisfying, safe and pleasurable sexual life (www.who.int/reproductivehealth/gender/sexual_health.html).

Despite the increased global attention to reproductive health in the last two decades, adolescent pregnancy is a growing source of alarm in many countries (ÏCAF, Ï994). The United Nations General Assembly in July, 1997 affirmed its commitment to make reproductive health universally available by the year 2015. Earlier in 1994, the UN Conference on population and development held in Cairo, Egypt prioritized the youth for the last decade. Moreover, many non-governmental organizations and governments had stepped up their advocacy and actions in reproductive health matters, and programmes were being introduced in many countries of Africa. Yet, the adolescents tend to be disproportionately negatively affected by poor reproductive health programmes. Young people all over the world are growing up in an increasingly complex environment that requires them to take tougher decisions, often without adequate preparation. Although it is generally known that the period of adolescence is a phase in life when young people are particularly vulnerable to many risks, especially in relation to their sexuality, they often lack access to adequate information, counseling and services on issues crucial to their development needs (Isiugo-Abanihe, 2005). A large proportion of young persons are in their most impressionist years when behaviours and character traits have not been fully formed; they reach sexual maturity before they develop mental/emotional maturity and the social skills needed to appreciate the consequences of their sexual activity (Fee and Youssef, 1993). Evidence of unmet need is reflected in research that confirms that some young people have poor understanding of the reproductive process, others harbour misconceptions such as the belief that pregnancy cannot occur during first sexual episode, and that use of contraceptives can cause infertility (Amazigo et al, 1998; ARFH, 2004; FMOH, 2003). One of the consequences of the involvement of young persons in risky sexual activities is that this group is disproportionately affected by reproductive morbidity including STI/HIV, unwanted pregnancies and their complications (Archibong, 1991; Brabin et al, 1995; Ekweozor et al, 1995; Bello etal, 1997; Arowojolu et al, 2003).

Of the peculiar reproductive health problems of the adolescents in Africa, the girl-child is most at risk. In Nigeria, like in many African countries, the young girl is naturally and socially disadvantaged to negotiate safer sex. This situation is even worse for the girl-child who lives in a culture where gender inequity is an accepted way of life, like in Northern Nigeria. The 2008 NDHS shows that $23 \%$ of women age 15-19 in Nigeria and $45 \%$ in North-Western zone are currently mothers or pregnant (NPC and ICF Macro, 2009). In fact, adolescent marriage and parenthood are a common sight in both urban and rural families in Northern Nigeria. One of the 2004 Nigerian National Population Policy objectives is increasing the integration of adolescents and young people into development efforts and effectively addressing their reproductive health and related needs. Thus, this study adopted qualitative approach in the investigation of the challenges adolescents' sexual and reproductive health in Northern Nigeria. 


\section{MATERIALS AND METHODS}

This study was located in North West geo-political zone of Nigeria. The zone consists of seven states, namely Jigawa, Kaduna, Katsina, Kano, Kebbi, Sokoto and Zamfara, and has an estimated population of $35,479,758$, which is about $25.9 \%$ of the national population of $137,794,744$ projected for mid-2006. The data used in this research article were collected between August and October, 2008 from a survey of three states in North-Western Nigeria, namely Kaduna, Kano and Katsina.

The study was a community based cross-sectional research, and the data collection instruments adopted included focus group discussions and in-depth interview guides. Focus group discussions (FGDs) were held with teenagers (both male and female) between the ages of 15 and 19. The goals of these FGDs were to collect information on the beliefs, attitudes, perceptions and views of participants so as to examine whether they perceived early marriage and childbearing to be issues that personally affect their lives as well as society in general; to increase our understanding of the impact of socio-cultural, environmental and proximate variables on adolescent reproductive behaviour; and to ascertain the prospects of change in behavior based on their responses. FGD was used in this research because of its ability to provide insights into complex patterns of sexual and reproductive behaviour and motivations, perceptions and attitudes of young people within defined rural and urban areas. Twelve FGDs were carried out in each of the states selected for the study. The groups were formed to be fairly homogenous with respect to residence (rural or urban), sex, marital and fertility status so as to minimize inhibitions in the flow of the discussions. Equal number of FGDs was designed for male and female adolescents because of the need to know independently the gender differences on the issues under consideration. Each FGD comprised six to ten discussants. Therefore, for each state, there were six FGDs in rural and six in urban LGAs. In each residence type (rural and urban), FGDs were conducted among the single (male and female), the married (male and female), and the adolescents of parental status (father and mother). A total of thirty-six (36) FGDs were conducted for this research; eighteen came from urban areas, while the other eighteen were conducted in rural areas.

Participants were chosen in a non-probabilistic random fashion from various neighborhoods or settlements to ensure a broad range of experiences within each group. Focus group discussions were led by trained indigenous moderators and note-takers (males and females depending on the sex of the group); most of whom were university undergraduates of humanity-based disciplines who had received training in focus group methodology. As a prelude to the discussion, the moderator provided a general introduction to the study and the purpose of bringing the participants together. Anonymity and confidentiality were assured, and permission (with explanation) sought for the use of tape recorder. Discussion outlines with lead questions were prepared by the researcher as a guide for the focus group facilitators. It was translated into Hausa for some groups (especially those in the rural areas) who might feel more comfortable discussing these issues in their indigenous language. The FGD guide organized each group's discussion on the same set of topics and in the same order to facilitate comparison. However, the discussion did not always rigidly proceed in that order; because the participants were free to bring up whatever issues they chose in response to a particular question, the moderators were instructed to maintain sufficient flexibility in introducing the topics to achieve a free-flow of the discussion. The discussions were tape-recorded, and later transcribed and translated prior to analysis; in both urban and rural settings, the translations were checked by a second person. 
Furthermore, sixteen in-depth interviews (IDIs) were carried out in each state. These comprised eight each from rural and urban areas. The selection of key informants was governed by the need to capture certain variables that are considered important, such as marital and fertility status. Essentially, the IDI was intended to serve as a vital data collection tools to gather crucial, in-depth and firsthand knowledge about the motivations for early marriage and childbearing. Therefore, rural and urban respondents comprised four male and four females each of marital and fertility status. On the whole, forty-eight in-depth interviews were conducted for this research. In-depth interview guide which consisted of topic guides to ensure that important issues were not forgotten during the interview was prepared by the researcher for use by the interviewers. Any relevant issues not included in the guide were introduced in the discussions. The interviews were conducted both in English and Hausa languages depending on the interviewees' choice of language. All the interviews were conducted in a very relaxed, unrushed atmosphere and relevant notes were taken by the interviewers. Overall, 252 focus group participants and 48 key informants were involved in the FGDs and IDIs respectively. Analysis of the data followed two approaches, namely ethnographic summary and a systematic coding via content analysis to accommodate verbatim quotations.

\section{RESULTS AND DISCUSSION}

\section{1. Age at first marriage and childbearing}

Age at marriage is crucial factor in women's lives, not only because of its association with overall completed fertility and with the meaning and consequences of adolescent fertility, but also due to its relation with the status of women. Early marriage in fact is favoured in different contexts. Legal codes governing family law usually establish the minimum age at first marriage. In spite of the existing legal minimum ages for marriage however, more often than not these are not enforced, especially in the rural areas. The cultural definitions of acceptability usually have much more force in the resulting outcome, than those imposed by law and removed from the realities of the people to whom they are supposed to apply. In most of these areas, early marriages are usually performed without the informed consent of the girl and often involve important age differences with the spouse, one element of unequal power relations between the spouses and of difficulty for empowerment.

The discussion on participants' choice of the best age at marriage in their communities attracted opposing views. While some participants in nearly half of the groups felt that a girl is 'ripe' (mature and ready) for marriage and childbearing as soon as she starts menstruation which is at about 15 years or younger, others said 18 years and above would be their choice if they could decide the best age at marriage and childbearing in their communities, because at that age she would have finished at least secondary school and/or has learnt some handwork that can be a source of income in her husband's house, and she would have been physically and emotionally matured enough to take care of herself and children properly. Male participants and largely from rural areas were more likely than their female and urban counterparts to feel that a girl who has seen her first menses was matured enough for marriage and should be married out. Their reasons were largely religious and moral, particularly "compliance with religious teachings and prevention of immoral behaviour" among girls in their community. An urban-based adolescent father-member of the FGD with adolescents who are currently fathers summed up the arguments with some insights as follows: 
The best age for marriage and child bearing especially for a girl is 18 years and above; that is when she finished secondary school; at least she has acquired some knowledge and her level of awareness has increased and she can take care of herself and family well, also at that age she is mature enough to carry a pregnancy and deliver without much problem."

This line of reasoning was hotly contested by some discussants in several other groups on moral grounds. Their views were summed up by a rural-based male participant when he said: "any year a girl wants to marry, she can go ahead and do so rather than restricting her to certain age. What if she cannot remain chaste till that time? Will you be happy to see your daughters mess around with men in the name of waiting for a certain age before marriage even though she has suitors? This totally leaves much to be desired." [This comment was greeted with much applause]. However, the participants were undecided and hardly specific on the best age at marriage and childbearing for boys because many of them felt that the age at marriage for boys was largely determined by the wish or wherewithal of the boy and/or his family, to cater for his family since by culture they were supposed to be the breadwinners.

The discussants however affirmed that adolescents are becoming sexually active at younger ages, and elements of force in their first sexual experiences abound. Consequently, they are unprepared for the consequences of sex. More importantly, sex, and sexual matters with adolescents, cannot be publicly discussed in many cultural environments of Nigeria and parents/teachers are usually at a loss when confronted with such matters. In northern Nigeria, it is common to find young girls getting involved in street trading in cities, apparently to augment the family income.

Many of these girls become easy prey to dubious customers and suffer all kinds of child molestation. For this study, marriage was defined as any stable union, irrespective of whether the male partner has performed certain traditional rites or not. Thus, both formal and informal unions were taken to mean marriage because of the significant effects both have on childbearing. In any case and within Nigerian cultural contexts, marriage is a union between persons of opposite sexes which involves rights and obligations fixed by law and custom.

A common perception about teenage marriage and childbearing, particularly among government, development agents, international agencies and NGOs, is that the behavior is a problem and should be discouraged. Against the backdrop of this perception, adolescents were asked if they personally thought that teenage marriage and childbearing was a problem in their communities which should be discouraged. In the focus group discussions, there was no clear consensus on whether teenage marriage and childbearing was a problem and should be discouraged.

In more than half of the groups, participants stated that teenage marriage and childbearing was not a problem even though they agreed it was a common practice in their communities, particularly in the rural areas. Two female participants, one rural- and the other urban-based captured the general feelings of the group thus:

What do you expect from a girl that is not schooling? Since we are discouraged from schooling and what every girl is doing here is to marry early, you have to join them. And when you are married, pregnancy and childbearing should be expected anytime. To me, I feel it is right since our parents did the same and nothing happened to them negatively. 


\begin{abstract}
Marriage is of God, whether a girl marries early and gives birth or not if she is destined to face problem she will definitely do so. There are cases of older women who still have pregnancy and child birth complications, and if God wishes, a girl that marries early can deliver safely without any complications. All these are the wonders of God, so to me teenage marriage is not a serious problem because in another way it helps in maintaining sanity in the society since girls are so corrupt and cannot keep themselves pure these days.
\end{abstract}

In this study, all the focus group participants and key informants stressed the effects of strong social pressure from within their communities to marry and bear children. Specific family members were reported to be particularly insistent. Far from discouraging early marriage and childbearing, the dominant picture was of familial pressure in favour of it. Girls experienced most pressure from parents and grandparents to marry to avoid 'messing around' with men and bringing shame and disrespect to the family; and boys, too, were expected to prove to parents and relatives that they were responsible, however pressures on them were not as much as on girls. In particular, the focus group participants generally agreed that parents decide when a girl marries, though some families allow her to choose her husband herself especially if she is educated to at least secondary school level, and insisted on her choice of partner. One currently married female participant explained:

Our culture insists that the first marriage is decided by parents because the girl is very young in most cases and may not know who is good for her; more so if there are many suitors. So in that case, the father and/or his brothers or mother decide when and who she marries.

Generally, in the focus group discussions, the responses buttressed the fact that marriage and childbearing remain central in the lives of men and women in northern Nigeria. Since marriage, among other things, confers status on individuals, those who remain unmarried after their mates have done so are socially alienated and looked down upon. The trend toward arranged marriage (particularly among female adolescents), rather than individual mate selection, received the approval of most discussants and interviewees both in urban and rural areas. The overwhelming majority of the married adolescents (especially females) interviewed reported that their marriages were arranged by parents, and that even in the case of individual mate selection, the approval and blessing of parents were obligatory. Sense of vulnerability and fear clearly exist as a number of participants expressed lack courage to engage or confront appropriate authorities, including their parents with reproductive health issues. An urban-based male adolescent and a currently married female participant provided further insights and summed up the discussants' fear, which was also heard in most of other FGDs:

Even if teenage marriage and child bearing is a problem we can't speak against it openly, because our people use religious reasoning to do whatever they are doing; but let us encourage girl-child education, and by so doing early marriage will be discouraged in the long run. 


\begin{abstract}
Teenage marriage might not be right, but who are you to stand against your parents' wishes; they will curse or disown you, and every child in this environment seeks and desires parental blessings in everything, so even if it is displeasing to us to marry at teenage age, we still obey and comply. If we can find our way, our parents should allow us to acquire formal education, and with that we will find something doing to take care of ourselves and our children if the worst comes.
\end{abstract}

The discussants and key informants reported increased risks of obstetric problems with teenage marriage and childbirth. The very high incidence of vesico-vaginal fistula in northern Nigeria is directly associated with teenage marriage and childbirth. More so, the adolescent girl is more likely to die during pregnancy and childbirth compared to woman aged 20 years and above. Yet, this age-long cultural practice has survived largely as a learned norm. The young girls who are not married see themselves as failing in one respect, just as their parents may become desperate. Some studies have linked the eagerness to marry off young girls to family poverty (Ulin, 1992; James, 2010). While this may be the underlying factor, it is unlikely to be the case if the teenagers could see good career options well ahead and look up to new role models. Here again, educational empowerment of adolescents, fostering of social support institutions that are adolescents-friendly, are widely advocated options. These could be coupled to a policy of democratization of contraceptive information and use.

Thus, given the obvious high level of sexual activity and marriage by the young in northern Nigeria, it should be reasonable to accept the fact and equip the young with reproductive health information in the overall interest of the society. A major opening here is to introduce reproductive health education into the formal and informal school curricula (James, 2012).

\title{
3. 2. Knowledge, attitudes and practice of contraceptives
}

In view of the apparent impact that knowledge and attitudes towards contraceptives have on their use, the key informants were asked if they ever heard that there were methods used by married and unmarried persons to prevent, space or delay a pregnancy, and if they approve or disapprove using any of these methods. These questions were also raised and discussed at focus group sessions. Questions relating to use of methods of contraception were also asked. Indeed data on knowledge and use of contraceptive methods are central to demographic analysis particularly fertility phenomenon. Knowledge about available contraceptive methods is the first step toward the adoption of appropriate methods.

The vast majority of focus group participants said that the use of any contraceptive methods to prevent, space or delay pregnancy and childbearing was not a common practice in their communities. A great number of participants mainly in urban groups however, reported that they ever heard of contraceptive methods and many of them claimed they knew some types of contraceptive methods. Condom was the most common method cited by these participants, although pills and injectables were also frequently mentioned. Females were more likely than male participants to claim ignorance of any methods of contraceptive.

In all the group discussions and interviews, strong views were expressed, either in approval or in opposition and condemnation of contraception. While some focus group discussants and in-depth interview key informants opposed it in its entirety, others approved of it not only for the married but also for the single women.

The disapproval of contraception particularly for the single/unmarried persons was based largely on moral and health concerns, particularly the feelings that it would amount to an 
"encouragement of illicit and premarital sex, and by extension an encouragement of immorality." Indeed in Muslim dominated parts of northern Nigeria, issues relating to modern contraception or family planning always incite religious sentiments which are provided as backing for any argument against it.

In the discussions, the responses concerning contraception were relatively sex-specific. The female groups, particularly the single girls in urban areas mostly mentioned condoms and pills directly. Injectables were most mentioned by the groups of currently married girls and currently mothers. Withdrawal method was mentioned by only one single girl participant, perhaps the nature of the method contributed to its relatively low general recognition. More than half of the female groups made reference to abstinence and to "local herbs and charms." Without doubt, however, the most widely recognized method for avoidance, delay and spacing of pregnancy among teenage women in northwestern Nigeria was abstinence from sex and local herbs and charms.

Most of the young men also mentioned condoms, and only passing reference was made by a couple of single boys groups to "a pill that women can take to avoid becoming pregnant." Two groups of currently teenage fathers and one group of currently married boys made brief mention of "herbs and charms." Withdrawal method was mentioned by participants of a single boy group; again, reference to this method was greeted with laughter. All but one male group mentioned abstinence, and, as with female groups, the majority of all male group recognized abstinence as a method of contraception.

In all the focus groups, only a few currently married female participants reported that they ever used a method of contraception, particularly pills. The vast majority of participants claimed they had never used and were not currently using any methods.

The main reason participants gave for non use of contraceptive methods even though a few reported that they were not against it, was spousal and parental non acceptance. A currently married female participant said: "Yes I know there are methods like condoms, tablets and injections; I am not against their use but our parents and husbands are against them; and you know in this part of the country we mostly dance to the tune of our parents and husbands." Other reasons cited for non use of contraceptives included fear of side effects and religion.

One female participant summarized this fear: "Contraceptives are not common in our communities; I have never used any, our parents and community leaders are very seriously against it, our religion discourages it and we are told that it has some dangerous side-effects on users." Males were more likely to mention religion, and females were more likely to mention spousal non acceptance and side effects.

On the whole, while many male and female participants in all the groups expressed disapproval, the strongest and most focal condemnation and disapproval came from the males. The arguments echoed in almost all the groups were summed up by a currently married ruralbased male adolescent when he explained:

There is no basis both in our religion and Hausa culture for unmarried people to do family planning or use contraceptives; the act for which contraception or family planning ought to be suitable is clearly not available for unmarried people. Aside from this, in Islam an unmarried adulterer or fornicator runs the risk of being stoned to death in the act.

\section{3. Reproductive health information and services}

In another question, respondents were asked if they ever discussed sexual and reproductive health matters (i.e. sex, pregnancy, marriage, childbearing, family size, 
contraception/family planning) with any person, such as parents, relatives, spouse, medical personnel, imam/priest or any other person, or sought reproductive health information and services. Essentially, promoting spousal discussion of family planning has frequently been advocated as a viable policy tool for narrowing the gender gap in partners' fertility intentions in developing countries. The widespread assumption, then, is that discussion works to promote contraceptive use by increasing knowledge of partner's attitude. For adolescents, discussion of sexual and reproductive health matters may improve knowledge of family planning attitudes and perception towards family size. This could also be achieved through mechanisms other than discussion, such as through conversations with third parties. Because discussion has become a focal point for policy making, determining the validity of this assumption is crucial.

As expected, discussants and key informants who reside in urban areas were more likely than their counterparts in rural arrears, to discuss reproductive health matters, including the number of children to have in life time. This is partly a function of western education. Until recent years, traditional teachings and social practices including strict gender segregation for certain activities, taboos, and social disapproval of premarital sex and pregnancy, made it rare for teenagers to discuss sexual and reproductive health matters. However, urbanization has loosened those social practices, and in the process sexual behaviour among youths has become more extended and premarital teenage pregnancy more frequent. Increasing urbanization, modernization and education, together with exposure to western media have, therefore, had an important influence in the disappearance of certain taboos and practices, and led to a decline in traditional values and liberality in attitudes toward sexual matters, including discussion of reproductive health issues. Obviously, females had a more favorable disposition than males towards family size discussion with partners, a condition which suggests male conservatism regarding spousal communication and influence in family size determination.

Generally in Nigeria, seeking information in all matters concerning sex is culturally perceived as a sign of promiscuity. Though, sometimes, affordability is the obstacle, parental resistance is probably the greater obstacle to adolescents seeking information and services. In the absence of information, misinformation thrives. The rural populace is often less informed and studies have shown that the rural woman is not only hard to reach but much more difficult to change her attitudes which are ingrained in her culture (Nwobodo, 1997). These culturally imposed obstacles to the democratization of reproductive health information stand a better chance of being redressed by the inculcation of a carefully planned culture-compatible reproductive health education in the curriculum of primary and secondary schools; it can also be incorporated in the programmes of the many existing traditional training institutions. Programme planners should exploit such socio-cultural institutions as age-grades clubs, bridesgrooming homes and even the local places of worship. Many programmes on family planning campaigns got remarkable successes when using within existing institutions and social structures (ICAF/Passages, 1993).

\section{CONCLUSIONS}

Given the above scenario, an effective intervention strategy must be based on an understanding of adolescents' experiences within specific contexts. The cultural survival pressures on adolescents combined with a certain obsession or fear about their personal identities and places in society within and outside of their families are powerful factors that both motivate sexual and reproductive behaviour, and also affect their ability to practice preventive measures. Zabin and Kiragu (1998) had observed in sub-Saharan Africa that public 
attention tends not to focus on the potential dangers of immediate post pubertal childbearing among young married women, not because the dangers are not known, but because strong cultural, political, and religious barriers exist to acknowledging them openly and, above all, to addressing their root causes. In this study, adolescents clearly reported their powerlessness and lack of levirate to confront appropriate authorities including their parents less they incur the wrath of curse because they desire parental blessings in everything. Many of them were unable or unwilling to negotiate reproductive behaviour; ability to negotiate reproductive behaviour varies by educational status, but until intervention strategies are undertaken to tackle issues relating to empowerment, and access to resources, improved information and services, the much needed change in adolescent reproductive behaviour in northern Nigeria would be a mirage.

\section{References}

[1] Amazigo, U; Silva, N; Kaufman, J. and Obikeze, D.S. (1998) Sexual activity and contraceptive knowledge and use among in-school adolescents in Nigeria. International Family Planning Perspectives, 23(1): 28-33

[2] Archibong, E.I. (1991), Illegal induced abortion--a continuing problem. International Journal of Obstetrics and Gynaecology, 34:261-265

[3] Arowojolu, A.O; Ilesanmi, A.O; Roberts, O.A. and Okunola, M.A. (2003), Sexuality, contraceptive choice and AIDS awareness among Nigerian undergraduates. African Journal of Reproductive Health, 6 (2): 60-70

[4] Association for Reproductive and Family Health (ARFH) (2004). The baseline survey results of the new horizons project. Unpublished project report

[5] Bello CSE, Egah DZ, Okwori EE, Nwokedi, EE, Katung, PY, Zoakah, AI, Opajobi, SO, Ayeni, JA, Barau, C and Mafuyai S (1997), Sexually transmitted diseases (STD): six years experience in Jos Teaching Hospital. Nigerian Journal of Medicine, 6(3): 83-86.

[6] Brabin L, Kemp J, Obunge OK, Ikimalo J, Dolimore N, Odu N.N, Hart C.A, Briggs ND (1995) Reproductive tract infections and abortions among adolescent girls in rural Nigeria. Lancet, 345, 270-271

[7] Ekweozor C.C, Olaleye O. D, Tomori O, Saliu I, Essien EM, Bakare RA, Oni AA, Oyewo OO, Okesola AO and Oyemenen IN (1995), Clinical epidemiology of STD patients seen in Ibadan. African Journal of Medicine and Medical Sciences, 24: 321-7.

[8] Federal Ministry of Health (FMOH) (2003), National HIV/AIDS and Reproductive Health Survey (NARHS).

[9] Fee, D and Youssef N (1993) Young people, AIDS and STD prevention: Experiences of peer approaches in developing countries. Unpublished WHO report

[10] ICAF/passages (1994) Contraceptive options for teens, xiii (2): 1-6.

[11] ICAF/Passages. (1993) Counseling Teens, xii: 1-8.

[12] Isiugo-Abanihe, I. M. (2005) "Comments on Benefits of Sexuality Education for Young Persons in Nigeria". Discussant at Understanding Human Sexuality Seminar Series 3 organized by African Regional Sexuality Resource Centre, in Lagos, March 24. 
[13] James, G. (2012) "Education and Sexuality: Towards Addressing Adolescents' Reproductive Health Needs in Nigeria." Current Research Journal of Social Sciences 4(4): 285-293.

[14] James, G. (2010) "Socio-cultural Context of Adolescents' Motivation for Marriage and Childbearing in North-Western Nigeria: A Qualitative Approach." Current Research Journal of Social Sciences 2(5): 269-275.

[15] National Population Commission (NPC) [Nigeria] and ICF Macro (2009). Nigeria Demographic and Health Survey 2008. Abuja, Nigeria: National Population Commission and ICF Macro.

[16] Nwobodo, É. D. (1997) 'Cocktail of adolescent's sexuality problems in Nigeria', MWAN Conference Proceedings. Ckiitstia, Nigeria.

[17] Shane B. (1992) Family Planning saves lives, 3rd Edition. USA.

[18] Ulin P.R. (1992) 'African women and AIDS; Negotiating behaviour change.' Soc. Sci. Med. 34: 63.

[19] www.who.int/reproductive-health/gender/sexual_health html: Gender and Reproductive Rights.

[20] Zabin, L.S. and K. Kiragu, (1998) 'The health consequences of adolescent sexual and Fertility behavior in Sub-Saharan Africa' Studies in Family Planning 29(2): 210-232.

[21] Kabiru Ibrahim Yankuzo, International Letters of Social and Humanistic Sciences 4 (2014) 1-8. 